\title{
A meta-analysis of D1 versus D2 lymph node dissection
}

\author{
Rajini Seevaratnam $\cdot$ Alina Bocicariu $\cdot$ \\ Roberta Cardoso - Alyson Mahar • Alex Kiss • \\ Lucy Helyer · Calvin Law $\cdot$ Natalie Coburn
}

Received: 5 April 2011/Accepted: 19 October 2011/Published online: 3 December 2011

(C) The International Gastric Cancer Association and The Japanese Gastric Cancer Association 2011

\begin{abstract}
Background Surgery is the only curative treatment for patients with gastric cancer. However, the extent of lymph node dissection is still debated. Therefore, with the publication of newer trial results, we conducted an updated meta-analysis of D1 versus D2 randomized controlled trials comparing outcomes.

Methods Systematic searches were conducted using Medline, Embase, and the Cochrane Central Register of Controlled Trials from January 1, 1985, to December 31,
\end{abstract}

R. Seevaratnam and A. Bocicariu contributed equally to this manuscript.

Electronic supplementary material The online version of this article (doi:10.1007/s10120-011-0110-9) contains supplementary material, which is available to authorized users.

R. Seevaratnam - A. Bocicariu $\cdot$ R. Cardoso - A. Mahar .

N. Coburn

Sunnybrook Research Institute, Sunnybrook Health Sciences

Centre, Toronto, Canada

\section{A. Mahar}

Department of Community Health and Epidemiology,

Queen's University, Kingston, Canada

\section{A. Kiss}

Department of Research Design and Biostatistics,

Sunnybrook Health Sciences Centre, Toronto, Canada

\section{Helyer}

Division of General Surgery, Department of Surgery,

Dalhousie University, Halifax, Canada

\section{Law · N. Coburn $(\square)$}

Division of Surgical Oncology, Sunnybrook Health Sciences

Centre and Odette Cancer Centre, Suite T2-60,

2075 Bayview Ave., Toronto, ON M4N 3M5, Canada

e-mail: natalie.coburn@sunnybrook.ca
2010. Meta-analyses were performed using RevMan v5 software. Both short- and long-term outcomes were analyzed. Subgroup analyses of $\mathrm{T}$ stage and spleen/pancreas resection versus preservation were performed.

Results Outcomes of 5 randomized trials involving 1642 patients (845 D1, 797 D2) enrolled from 1982 to 2005 were included. Despite the addition of the more recent trials, overall hospital mortality and reoperation rates were still higher in D2 cases. Subgroup analysis of recent trials and spleen/pancreas preservation revealed no significant difference in hospital mortality between groups. Five-year overall survival was similar between D1 versus D2 trials. Sub-analysis by tumor depth and spleen/pancreas preservation detected trends for improved survival with D2 lymphadenectomy in T3/T4 patients and those with spleen/ pancreas preservation.

Conclusion Earlier trials show that D2 dissections have higher operative mortality, while recent trials have similar rates. A trend of improved survival exists among D2 patients who did not undergo resection of the spleen or pancreas, as well as for patients with T3/T4 cancers.

Keywords Lymph node dissection · D2 - D1 . Gastric cancer · Meta-analysis

\section{Introduction}

Currently, surgery is the mainstay of cure for gastric cancer patients in North America [1]. However, the type of curative resection and, in particular, the extent of lymph node dissection is debated. While D2 resection is considered the standard procedure in the East, many surgeons in the West continue to perform D1 resections, as randomized controlled trial (RCT) evidence to the contrary has been 
lacking. Patients with gastric cancer have a high frequency of regionalized disease with nodal metastasis; relapses after curative surgery due to local or regional lymph node metastasis are common. Local recurrence has been documented in up to $87.5 \%$ of patients presenting with relapse [2]; thus, an extended lymph node dissection is advocated by many surgeons to decrease the chance of local recurrence and increase the survival rate. The first RCT comparing D1 to D2 resections was performed between 1982 and 1986 in South Africa and consisted of only 43 patients [3]. Despite no operative mortality, the D2 group had greater operating time, more blood transfusion, longer hospital stay, and higher reoperation rates. However, 3-year survival was similar between groups [3]. Subsequently, the Medical Research Council (MRC) of UK trial (1986-1993) and the Dutch Gastric Cancer Trial (DGCT) (1989-1993) were launched, both of which were large-scale, multi-center RCTs that recruited a significant number of patients (400 and 711, respectively) [4, 5]. Both trials revealed similar short-term outcomes, including significantly higher postoperative stay, morbidity, and mortality rates with the D2 resection [4, 5]. Long-term outcomes failed to show any significant differences in 5-year survival between groups [6, 7]. An updated analysis of the Dutch trial has shown a decrease in the gastric cancer-related mortality in the D2 group on 15-year follow-up, but no statistically significant difference in overall survival [8]. In 1993, a Hong Kong trial (NCT00260884) was launched to compare D1 versus D3 lymphadenectomy [9, 10]; however, the definition that was used for D3 [11] is now considered D2 [12], essentially making this trial an RCT of D1 versus D2. Although no deaths were reported during the perioperative period for either the D1 or D2 arms, the morbidity rate was significantly higher and hospital stay was significantly longer for the extended lymph node dissection group [9]. Furthermore, follow-up survival analysis revealed that patients in the D2 group had a significantly longer 5-year overall survival compared to D1 patients [10]. More recent trials, the British/Edwards (1996-2002) and Italian Gastric Cancer Study Group (IGCSG)-R01 (1998-2005), demonstrated that short-term morbidity and mortality were similar between D1 and D2 groups, and that the complication rate following D2 dissections was much lower than previously published rates [13, 14]. Furthermore, the British/Edwards trial revealed a significant 5-year survival benefit for patients undergoing D2 compared to D1 [13]. This survival benefit was found particularly beneficial for patients with T3 tumors, N0 and N1 nodal disease, stage III cancers, cardia tumors, and those who had their spleen preserved [13]. However, the randomization to treatment for the Edwards trial was by postal code, introducing significant confounding [13].
These updated results suggested that a re-evaluation of the short- and long-term outcomes of the D1 and D2 surgical procedures was needed. The goal of this study was to perform an updated meta-analysis with the addition of the most recent trial results.

\section{Methods}

Data sources

Electronic literature searches were conducted using Medline and Embase from January 1, 1985, to December 31, 2010, according to the search algorithm presented in Appendix A in the Supplementary material. Search terms included: [exp Stomach Cancer/or (((gastric or stomach) adj1 cancer\$) or ((gastric or stomach) adj1 carcinoma) or ((gastric or stomach) adj1 adenocarcinoma) or ((gastric or stomach) adj1 neoplasm\$)).mp.] and [exp Lymphadenectomy/or lymph node/or (D1 or D2).mp [mp = title, abstract, subject headings, heading word, drug trade name, original title, device manufacturer, drug manufacturer name] or cancer staging/] and [clinical trial/or controlled clinical trial/or exp comparative study/or meta analysis/or multicenter study/or exp practice guideline/or randomized controlled trial/] not [Case Report/or review]. A separate search of the Cochrane Central Register of Controlled Trials (1985-2010) was performed using the search term "gastric cancer". No attempt was made to locate unpublished material.

\section{Study selection and review process}

To be eligible, studies had to meet the following criteria: (1) RCT investigating D1 versus D2 lymph node dissection in patients with gastric adenocarcinoma; (2) reported shortor long-term outcomes of trial; (3) patients had a pathological examination of the surgical specimens and lymph nodes dissected; and (4) published in peer-reviewed journals in English. Studies were excluded according to the following criteria: (1) randomized to D3 or D4 lymph node dissection groups; (2) studies that did not provide short- or long-term trial outcomes; (3) studies that involved animals and/or ex vivo samples; (4) studies that involved patients with mixed cancer with no separate analysis of gastric cancer subjects; and (5) review articles, meta-analyses, abstracts, conference proceedings, editorials/letters, and case reports. All electronic search titles, selected abstracts, and full-text articles were independently reviewed by a minimum of two reviewers (NC, LH, RS, RC). Disagreements on study inclusion/exclusion were resolved with a consensus meeting. 
Data extraction and analysis

A systematic approach to data extraction was used to produce a descriptive summary of participants, interventions, and study findings. The first reviewer (AB) independently extracted the data and a second reviewer (RS) checked the data extraction. No attempt was made to contact authors for additional information. The Consort 2010 checklist was used to evaluate the quality of RCTs (Appendix B in the Supplementary material [15]). The Consort checklist consists of 23 essential items that should be included in RCTs, as well as a diagram for documenting the flow of participants through the trial [15].

Statistical analyses were conducted using the RevMan version 5 software package (http://ims.cochrane.org/ revman, Cochrane Collaboration, Oxford, UK). The relative risk ratio (RR) and $95 \%$ confidence interval $(\mathrm{CI})$ were calculated for short-term outcomes, and odds ratios (ORs) and $95 \%$ CIs were calculated for survival. We assessed the heterogeneity between studies by using the $I^{2}$ test, which indicates the presence of heterogeneity when $I^{2} \geq 50 \%$ $[16,17]$. In different subgroup analyses, we found values for $I^{2}$ of both $<$ and $\geq 50 \%$. As a result, considering the diversity in the trials (location, physicians, training, surgical technique, etc.), we selected the random effects model for all analyses [16, 17]. Studies were weighted according to the inverse variance method of DerSimonianLaird [16, 17]. Statistical significance was considered when $p<0.05$.

\section{Results}

Literature search

A total of 3608 titles/abstracts were identified from the electronic searches and reference lists for preliminary review. After removal of duplicates and screening for relevant titles and abstracts, 112 articles were identified as pertaining to lymph node dissection, and of these 55 were submitted for a full-text review. Five randomized clinical trials and a total of 9 articles [3-7, 9, 10, 14, 18] involving 1642 patients met our inclusion criteria and were included in this review (Fig. 1). The descriptive characteristics of each included study are presented in Table 1. Randomization procedure and allocation concealment were adequate for the five trials.

\section{Short-term outcomes}

Overall hospital mortality for all 5 trials was significantly higher for D2 patients $(7.5 \%)$ than for D1 patients $(3.8 \%)$, with an RR of 2.02 (95\% CI 1.30-3.14, $p=0.002)$ (Fig. 2c). To demonstrate the change in hospital mortality

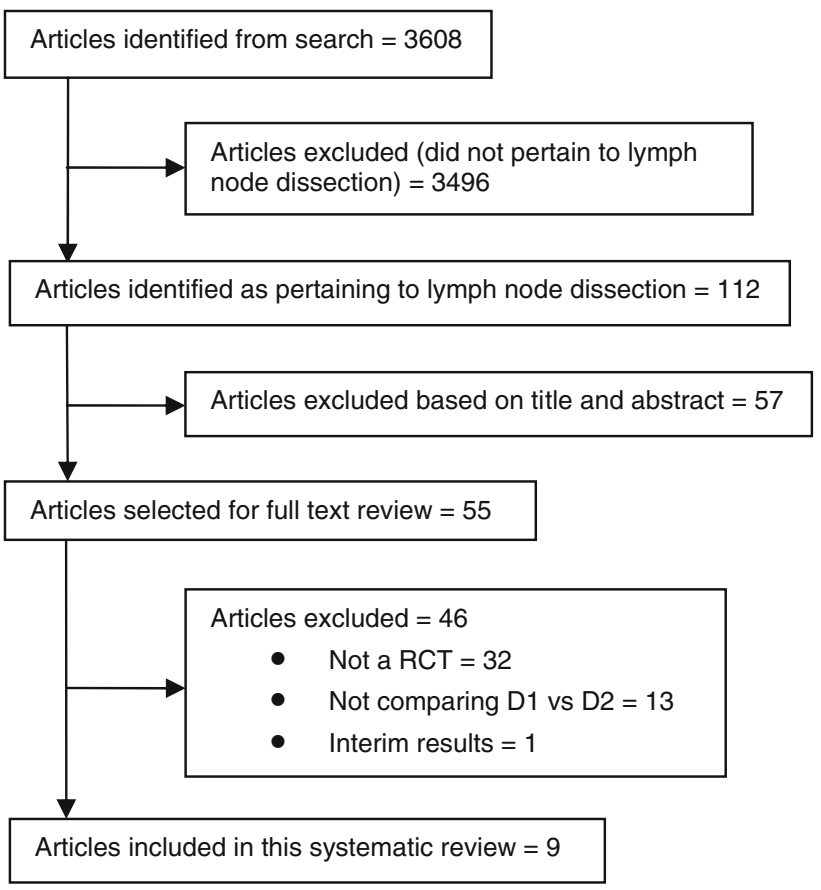

Fig. 1 Article selection flow. $R C T$ randomized controlled trial

over time, we performed a sub-analysis dividing studies into early trials [3-5] and recent trials [9, 14]. Hospital mortality was significantly greater for D2 patients $(10.5 \%)$ compared to D1 patients $(4.6 \%)$ in early trials, with an RR of 2.23 (95\% CI 1.44-3.44, $p=0.0003$ ) (Fig. 2a). However, in recent trials, hospital mortality was no longer significantly different between groups (D1: $1.5 \%$ vs. D2: $1.2 \%$, RR $0.74,95 \%$ CI $0.17-3.26, p=0.70$, Fig. 2 b), with much lower mortality rates being reported for the D2 procedure compared to earlier studies (1.2 vs. $10.5 \%$, respectively). To elucidate whether the difference in overall hospital mortality was due to spleen and/or pancreas removal, we performed a subgroup analysis of data from 2 trials $[4,5]$, which reported results for resection and preservation of these organs (Fig. 3). Hospital mortality was no longer significantly higher in the D2 group when comparing patients with spleen/pancreas preservation (D1: $4.0 \%$ vs. D2: $5.9 \%, p=0.25$ ) (Fig. 3c). Hospital mortality rates among patients with spleen resection (D1: $8.2 \%$ vs. D2: $27.3 \%, p=0.23$, Fig. $3 \mathrm{a}$ ), as well as the rates among those with spleen and pancreas resection (D1: $16.7 \%$ vs. D2: $13.7 \%, p=0.60$, Fig. $3 \mathrm{~b}$ ) were similar. To further clarify whether D2 resections had a higher hospital mortality because of spleen or spleen and pancreas removal, we performed a subgroup analysis of only D2 patients reported in 2 studies [4, 5] (Fig. 4). Patients with spleen preservation had significantly lower hospital mortality than those who had their spleen resected (5.7 vs. $27.3 \%$, respectively, RR $0.21,95 \%$ CI $0.10-0.43, p<0.0001$ ) (Fig. 4a). However, a significant difference was not found between 
Table 1 Study Characteristics of the 5 included Randomized Lymph Node Dissection Trials

\begin{tabular}{|c|c|c|c|c|c|c|c|c|c|c|c|}
\hline \multirow[t]{2}{*}{ Trial Name } & \multirow[t]{2}{*}{ Design } & \multirow{2}{*}{$\begin{array}{l}\text { Study } \\
\text { Years }\end{array}$} & \multirow{2}{*}{$\begin{array}{l}\text { Patients } \\
(\mathrm{N})\end{array}$} & \multirow[t]{2}{*}{ Age $(y)^{\mathrm{a}}$} & \multicolumn{2}{|c|}{ Gender } & \multicolumn{4}{|c|}{ Tumor stage } & \multirow{2}{*}{$\begin{array}{l}\text { Additional } \\
\text { organs removed }\end{array}$} \\
\hline & & & & & M & $\mathrm{F}$ & $\mathrm{T} 1$ & $\mathrm{~T} 2$ & $\mathrm{~T} 3$ & $\mathrm{~T} 4$ & \\
\hline \multirow{2}{*}{$\begin{array}{l}\text { South African } \\
\text { Trial [3] }\end{array}$} & \multirow[t]{2}{*}{ RCT (R1 vs R2) } & \multirow[t]{2}{*}{ 1982-1986 } & D1: 22 & $45 \pm 8.9$ & 12 & 10 & 6 & 5 & 11 & 0 & NR \\
\hline & & & D2: 21 & $55.8 \pm 11.4$ & 15 & 6 & 7 & 5 & 9 & 0 & NR \\
\hline \multirow{3}{*}{$\begin{array}{l}\text { Dutch } \\
\text { Trial }[5,7,18]\end{array}$} & \multirow[t]{3}{*}{ RCT (D1 vs D2) } & \multirow[t]{3}{*}{$1989-1993$} & D1:380 & $65(31-84)$ & 216 & 164 & $98^{\mathrm{b}}$ & $181^{\mathrm{b}}$ & $94^{\mathrm{b}}$ & $0^{\mathrm{b}}$ & $S^{f}: 41(11 \%)$ \\
\hline & & & & & & & & & & & $\mathrm{DP}^{\mathrm{f}}: 10(3 \%)$ \\
\hline & & & D2:331 & $63(21-84)$ & 188 & 143 & $85^{\mathrm{c}}$ & $152^{\mathrm{c}}$ & $82^{c}$ & $0^{\mathrm{c}}$ & $\begin{array}{l}S^{f}: 124(38 \%) \\
D^{f}: 98(30 \%)\end{array}$ \\
\hline \multirow{3}{*}{$\begin{array}{c}\text { MRC }(\mathrm{UK}) \\
\text { Trial }[4,6]\end{array}$} & \multirow[t]{3}{*}{ RCT (D1 vs D2) } & \multirow[t]{3}{*}{ 1986-1993 } & D1:200 & $67(38-86)$ & 132 & 68 & $48^{\mathrm{b}}$ & $63^{\mathrm{b}}$ & $84^{\mathrm{b}}$ & $0^{\mathrm{b}}$ & S: $54(27 \%)$ \\
\hline & & & & & & & & & & & S + DP: $8(4 \%)$ \\
\hline & & & D2:200 & $67(26-83)$ & 138 & 62 & $40^{\mathrm{b}}$ & $69^{\mathrm{b}}$ & $86^{\mathrm{b}}$ & $0^{\mathrm{b}}$ & $\begin{array}{l}\text { S: } 18(9 \%) \\
\text { S + DP: } 113(57 \%)\end{array}$ \\
\hline \multirow{4}{*}{$\begin{array}{l}\text { Hong Kong } \\
\text { Trial }[9,10]\end{array}$} & \multirow[t]{4}{*}{$\mathrm{RCT}\left(\mathrm{D} 1\right.$ vs $\mathrm{D}^{\mathrm{g}}$ ) } & \multirow[t]{4}{*}{ 1993-1999 } & D1:110 & $68(27-77)$ & 84 & 26 & 23 & 26 & 56 & 5 & S: $3(3 \%)$ \\
\hline & & & & & & & & & & & S + DP: $1(1 \%)$ \\
\hline & & & D3 ${ }^{\mathrm{g}}: 111$ & $67(23-76)$ & 86 & 25 & 29 & 20 & 59 & 3 & S: $1(1 \%)$ \\
\hline & & & & & & & & & & & S + DP: $13(12 \%)$ \\
\hline \multirow{4}{*}{$\begin{array}{l}\text { IGCSG R01 } \\
\text { (Italy) [14] }\end{array}$} & \multirow[t]{4}{*}{ RCT (D1 vs D2) } & \multirow[t]{4}{*}{ 1998-2005 } & D1:133 & $63.5(30-81)$ & 67 & 66 & $49^{\mathrm{d}}$ & $42^{\mathrm{d}}$ & $40^{\mathrm{d}}$ & $0^{\mathrm{d}}$ & S: $9(7 \%)$ \\
\hline & & & & & & & & & & & S + DP: $2(1.5 \%)$ \\
\hline & & & D2:134 & $62(22-87)$ & 64 & 70 & $39^{\mathrm{e}}$ & $55^{\mathrm{e}}$ & $37^{\mathrm{e}}$ & $0^{\mathrm{e}}$ & S: $12(9 \%)$ \\
\hline & & & & & & & & & & & S + DP: $2(1.5 \%)$ \\
\hline
\end{tabular}

$\mathrm{MRC}=$ Medical Research Council; IGCSG = Italian Gastric Cancer Study Group; RCT = randomized control trial; $\mathrm{N}=$ number; $\mathrm{M}=$ male; $\mathrm{F}=$ female; $\mathrm{NR}=$ not reported; $\mathrm{S}=$ spleen; $\mathrm{DP}=$ distal pancreas; $\mathrm{P}=$ pancreas

$\mathrm{a}=$ age reported as mean $\pm \mathrm{SD}$ or median with range; $\mathrm{b}=5$ patient have been omitted; $\mathrm{c}=12$ patients have been omitted; $\mathrm{d}=2$ patients unknown $\mathrm{T}$ stage; $\mathrm{e}=3$ patients unknown $\mathrm{T}$ stage; $\mathrm{f}=$ data missing for some patients; $\mathrm{g}=$ Published as D3, however protocol is actually D2

patients who had their spleen and pancreas resected versus those with preservation of these organs (6.8 vs. $13.7 \%$, respectively, RR $0.63,95 \%$ CI $0.13-3.11, p=0.57$, Fig. 4b). Nonetheless, combining all patients who had either of these organs preserved showed significantly lower hospital mortality compared to all patients who had either of these organs resected in the D2 group (5.9 vs. $16.1 \%$, respectively, RR 0.34 , 95\% CI $0.13-0.86, p=0.02$ ) (Fig. 4c).

The reoperation rate was analyzed in 4 studies $[3,5,9$, 14] and showed a significantly higher rate in D2 patients (11.4\%) compared to D1 patients $(5.1 \%)$, with an RR of 2.24 (95\% CI 1.52-3.32, $p<0.0001$ ) (Fig. 5). Organ resection/preservation sub-analysis in this patient population could not be performed as information was not published.

\section{Long-term outcomes}

The overall 5-year survival rate was reported in 3 studies $[6,7,10]$ and showed similar results for D1 (43.5\%) and D2 $(44.9 \%)$ patients (OR 1.06, 95\% CI 0.85-1.33, $p=0.58$, Fig. 6). To reveal whether patients with T1/T2 or T3/T4 tumors had a survival difference with respect to their dissection group, we performed a subgroup analysis by depth of tumor invasion in the 2 studies that reported this outcome [6, 7]. No difference was found for T1/T2 patients (D1: $55.4 \%$ vs. D2: $52.3 \%$, OR 0.86 , 95\% CI $0.59-1.27$, $p=0.46$, Fig. 7a), although a trend was detected for T3/T4 patients (D1: $13.5 \%$ vs. D2: $19.5 \%$, OR 1.59, 95\% CI $0.89-2.84, p=0.11$, Fig. 7b). A subgroup analysis by nodal status to elucidate whether node-negative or nodepositive patients had a survival difference with respect to their dissection group could not be performed as only 1 study reported values [6]. Finally, to determine the effect of spleen/pancreas preservation on survival, we performed a sub-analysis using the data provided from 2 trials [6, 10] (Fig. 8). The spleen/pancreas preservation subgroup had a trend for 5-year survival benefiting D2 compared to D1 patients (54.9 vs. $43.0 \%$, respectively), with an OR of 1.46 (95\% CI 0.98-2.19, $p=0.07$ ) (Fig. 8).

\section{Discussion}

The extent of lymphadenectomy continues to be a controversial topic. Previous reviews and meta-analyses have tried to clarify the debate [19-24]. With the publications of 


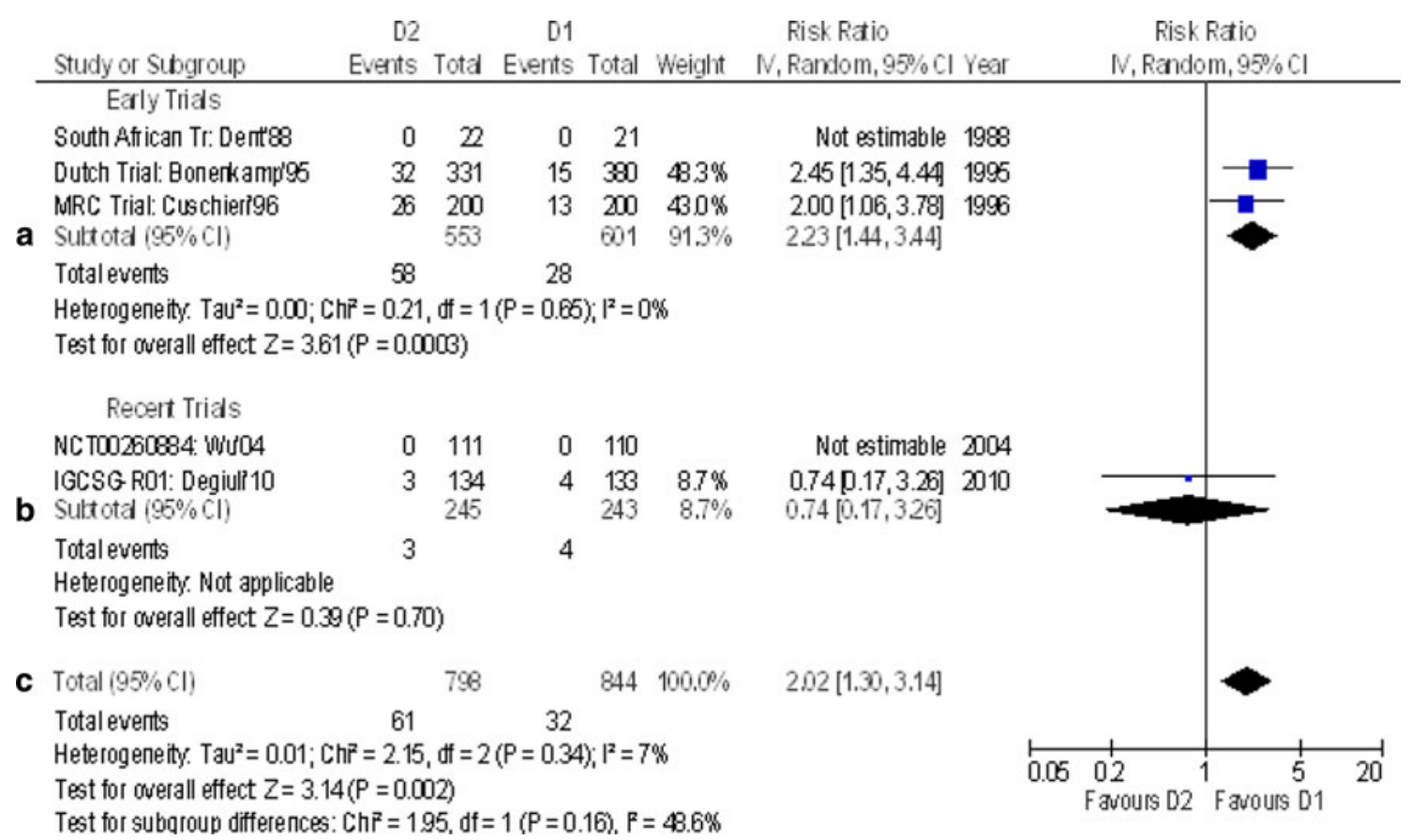

Fig. 2 Hospital mortality for D1 versus D2 randomized controlled trials according to early trials (a), recent trials (b) and overall total for all trials (c). Total hospital mortality (c) and subtotal hospital mortality for early trials (a) were significantly higher in the D2 group

the short-term outcomes of the most recent trial (IGCSGR01) and the 15-year follow-up of the Dutch trial [8], both of which reveal favorable results for the $\mathrm{D} 2$ resection, we sought to perform an updated meta-analysis of all RCTs comparing D1 and D2 procedures.

\section{Short-term outcomes}

Despite the addition of the most recent IGCSG-R01 trial, which found no significant difference in hospital mortality between D1 (3\%) and D2 (2.2\%) resections [14], total hospital mortality (combining all trials) was still significantly higher in the D2 group (Fig. 2c). This lack of change in the results is likely due to the number of patients enrolled in the recent trials (D1: 133 and 36, D2: 134 and 82, respectively) being too small to overpower the difference in mortality rates found in the earlier trials [3-5]. Indeed, in a separate power analysis (results not shown), we found that the IGCSG-R01 trial would need to add 150 more patients to each arm in order for hospital mortality to become statistically non-significant $(p=0.06)$ in a meta-analysis (such as the one presented in Fig. 2c), when combined with the much larger MRC and Dutch trials. Similarly, despite the lack of significance in reoperation rates in the IGCSG-R01 trial between D1 (2.2\%) and D2 (3.0\%) resections [14], the relatively low patient enrollment could not negate the
( $p=0.002$ and $p=0.0003$, respectively). Hospital mortality for recent trials $(\mathbf{b})$ was not significantly $(p=0.70)$ different between D1 and D2 groups. CI confidence interval, MRC Medical Research Council, IGCSG Italian Gastric Cancer Study Group

higher reoperation rates found in the Dutch Trial [5] (Fig. 5). Prior meta-analyses have all reported higher hospital mortality and reoperation rates for the D2 procedure [19-21, 23, 24] and have thus stated that D2 dissections are not advantageous. Our meta-analysis differs from those previously published as it includes the completed short-term results of the IGCSG-R01 trial. The recently published meta-analysis by Memon et al. [23] only considered the interim analysis of the IGCSG-R01 trial [25]. Additionally, our meta-analysis does not include the D1 versus D3 trial reported by Robertson et al. [26], which describes dissection around the celiac axis and para-aortic nodes as well as complete removal of the spleen and distal pancreas for D3 patients. This trial was combined with the other D1 versus D2 trials in the meta-analyses performed by Lustosa et al., Yang et al., and Memon et al. [20, 21, 23], which clouds the issue of D1 versus D2 dissection. Most importantly, none of the earlier meta-analyses examined the considerable effect of advances in surgical techniques and perioperative care with time. Our sub-analysis by trial year clearly demonstrated that while older trials favored D1 (Fig. 2a), both newer trials $[13,14]$ showed no significant differences in hospital mortality between D1 and D2 resections (Fig. 2b), suggesting that the D2 procedure may be performed without significantly higher postoperative risks. The observed improved mortality rates associated with D2 


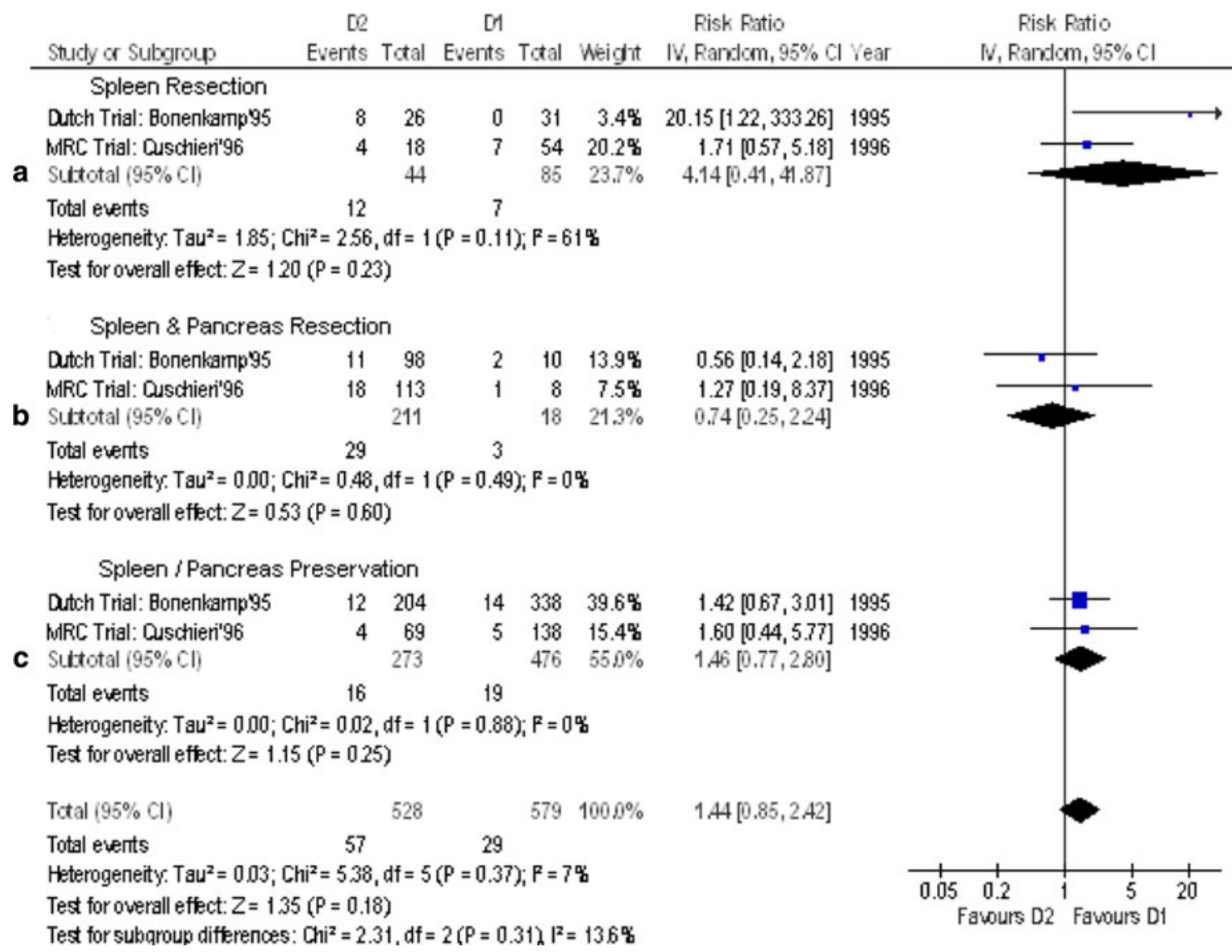

Fig. 3 Hospital mortality for D1 versus D2 randomized controlled trials according to spleen resection (a), spleen and pancreas resection (b), and pancreas and/or spleen preservation (c). Hospital mortality was not significantly different between D1 and D2 procedures for all subgroups ( $p=0.23, p=0.60$, and $p=0.25$, respectively)

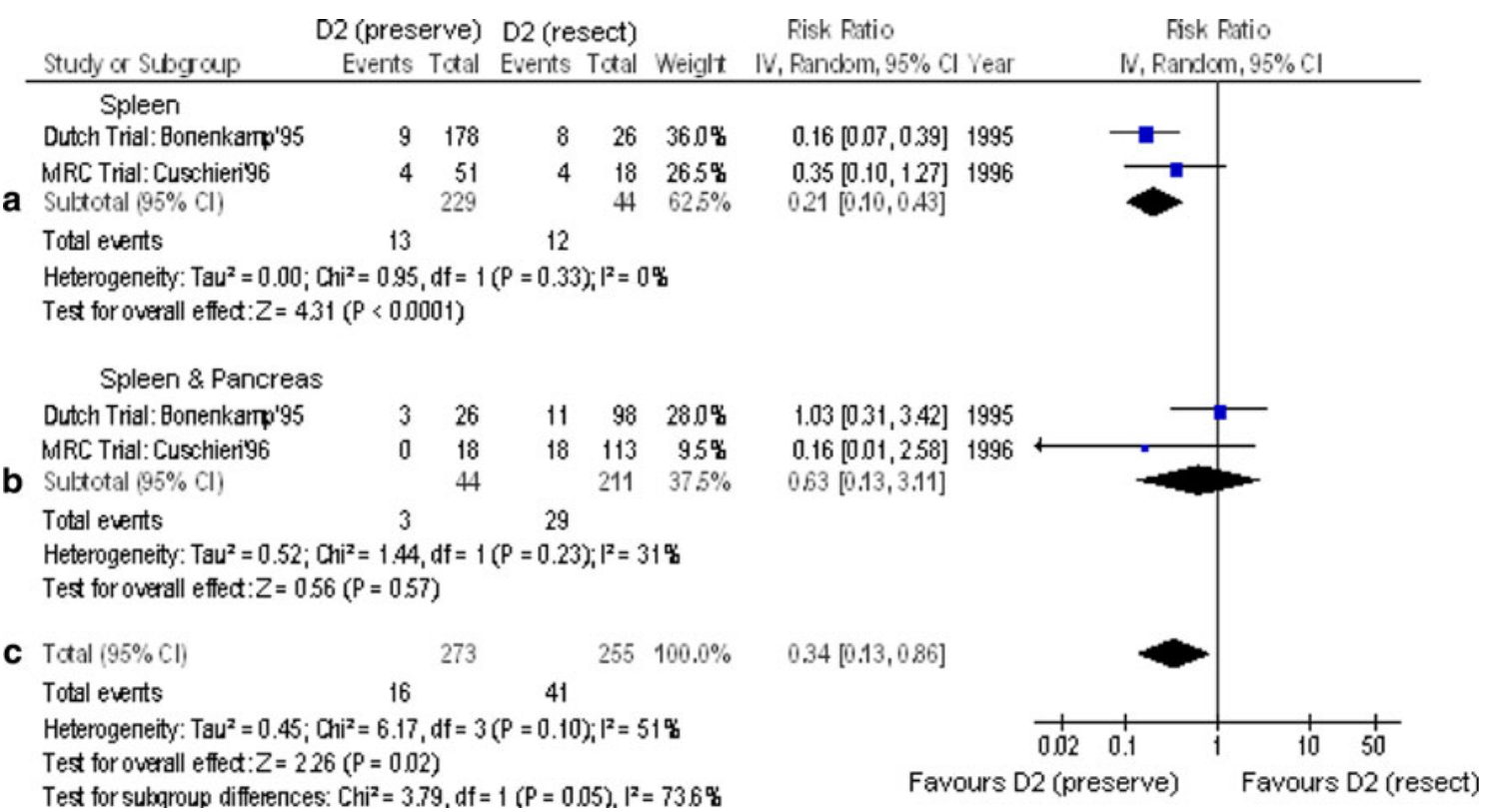

Fig. 4 Hospital mortality for patients allocated to the D2 procedure. Spleen and/or pancreas resection (D2 resect) was compared to spleen and/or pancreas preservation (D2 preserve) among D2 patients only. Patients with spleen preservation (a) had significantly lower mortality compared to their resection counterparts $(p<0.00001)$. Patients with different mortality rates compared to their resection counterparts. However, the total preservation group (c) had significantly lower mortality compared to the total resection group $(p=0.02)$ spleen and pancreas preservation (b) did not have significantly 


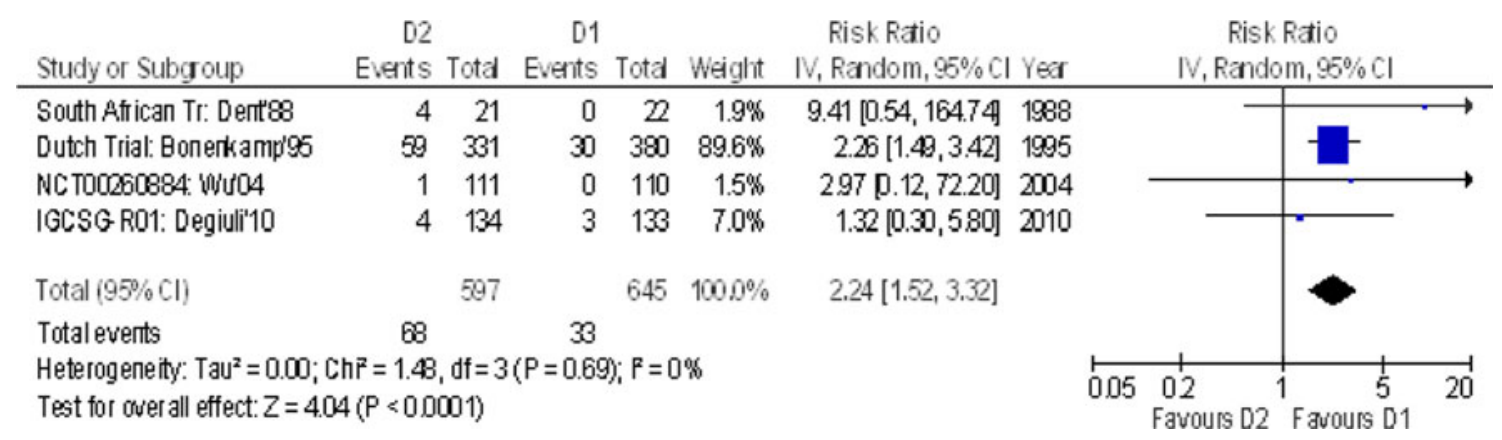

Fig. 5 Reoperation rates for D1 versus D2 randomized controlled trials. The combined total reoperation rate was significantly higher for the D2 group $(p<0.0001)$

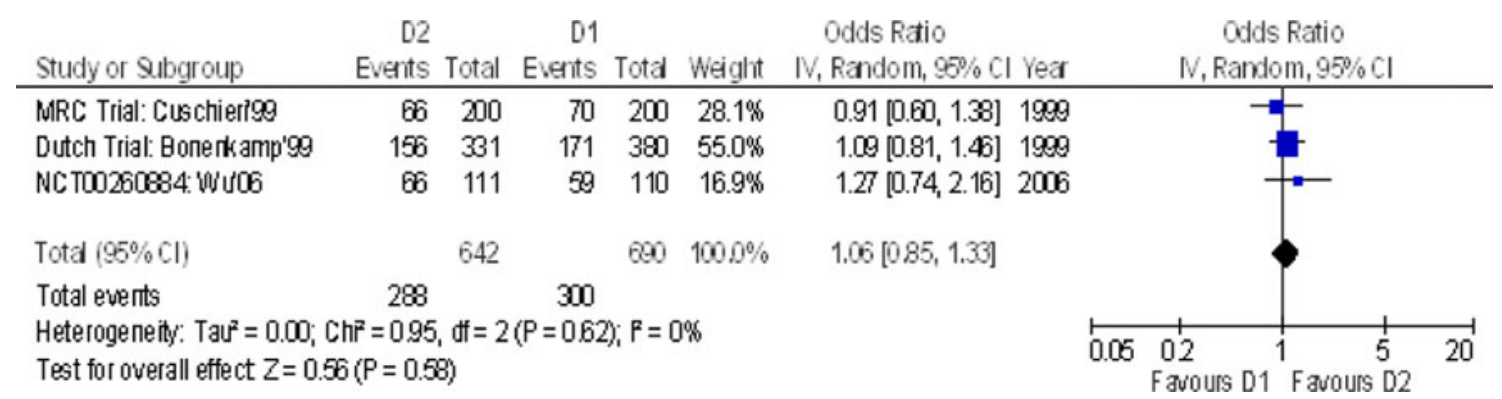

Fig. 6 Overall 5-year survival for D1 versus D2 randomized controlled trials. The combined total survival rate was not significantly different between groups $(p=0.58)$

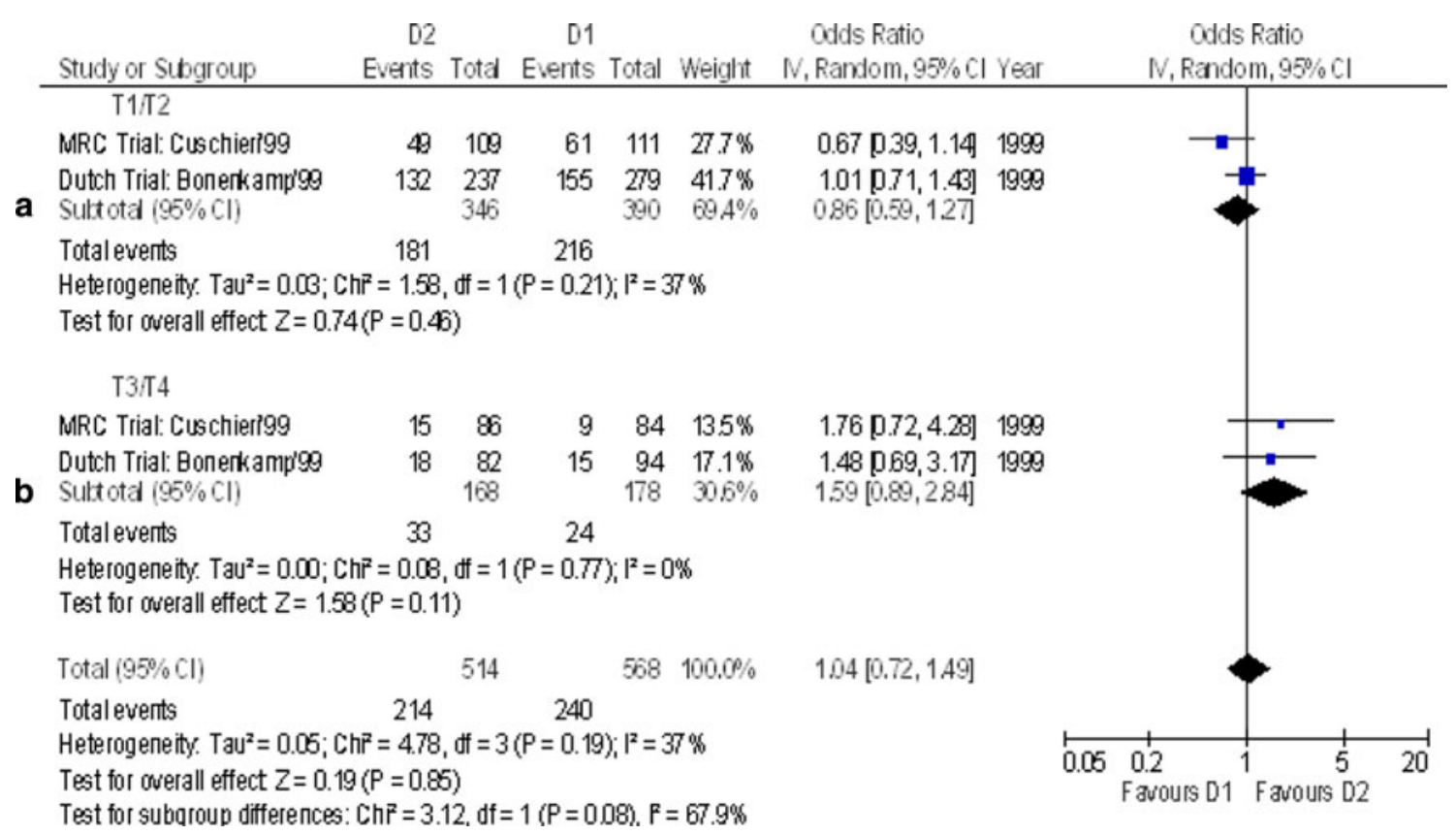

Fig. 7 Overall 5-year survival according to patients staged as T1/T2 (a) and T3/T4 (b). Patients in the D2 group who were staged as T3 or T4 had a trend for longer survival $(p=0.11)$ than those in the D1

dissection were likely also affected by more rigorous patient selection, increased training, and centralization of this type of surgery in high-volume hospitals [14]. group. Survival was not significantly different between D1 and D2 groups for patients staged as T1 or T2 $(p=0.46)$

However, the most noteworthy factor in patient outcomes is likely a change in the surgical protocol with regards to resection of the spleen and distal pancreas. 


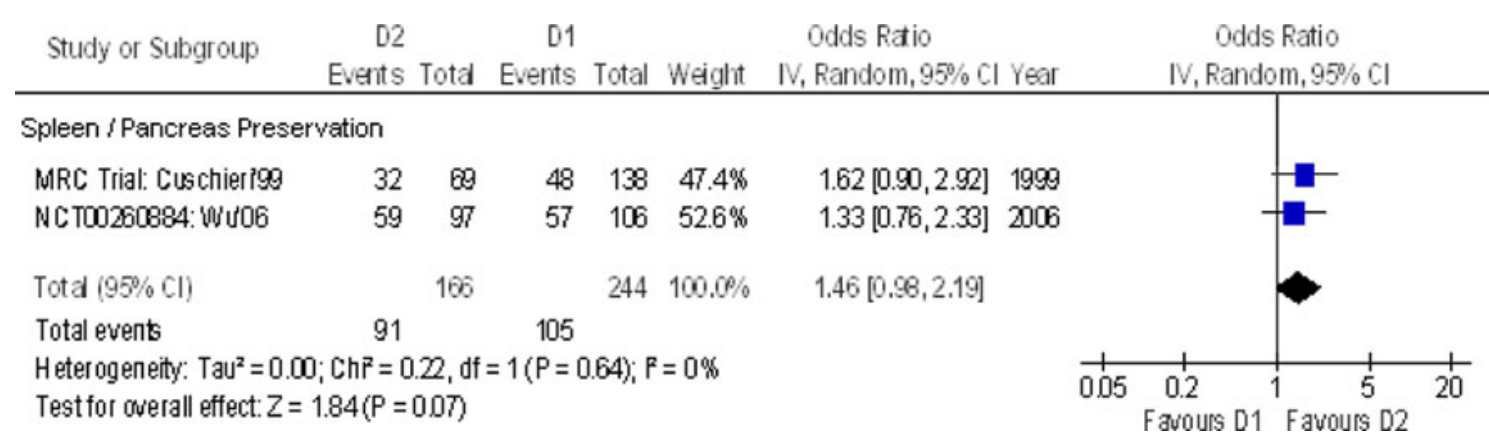

Fig. 8 Overall 5-year survival according to spleen/pancreas preservation. Patients in the D2 group with pancreas and/or spleen preservation had a trend for a longer survival rate $(p=0.07)$ compared to those in the D1 group

Organ resection versus organ preservation

The well-recognized drawback of the D2 procedure in the earlier trials was the necessary resection of the spleen and pancreatic tail. The South African [3], Dutch [5, 7, 18], and MRC [4, 6] trials required resection of the spleen and pancreatic tail in order to adequately remove the D2 lymph node stations 10 and 11 in proximal tumors, whereas those organs were removed in D1 resections only in cases of tumor invasion. In the Hong Kong trial [9, 10], combined organ (en-bloc) resection involving a hemipancreaticosplenectomy or a total gastrectomy, or both, was performed to facilitate nodal dissection in patients with lymph node metastases along the splenic artery (station 11) or in the right paracardia region (station 1). However, in the IGCSG-R01 trial, there was a modification of a D2 resection whereby splenectomy and spleno-pancreatectomy were not considered part of the routine procedure [14]. Instead, in the IGCSG-R01 trial, the spleen was removed according to the Maruyama technique only when the tumor was in the left part of the upper stomach or located close to the greater curvature, beyond Demel's point [14, 27].

As mentioned above, when all patients were combined, there was a significant increase in hospital mortality for D2 patients compared to D1 patients (Fig. 2c), a finding which has been published repeatedly in previous meta-analyses [19-21, 23, 24]. However, none of those analyses have explored the effect of spleen and/or pancreas resection. Our focused evaluation of D1 and D2 resections with spleen/ pancreas preservation showed hospital mortality was no longer significantly different between groups (Fig. 3c). Moreover, when comparing hospital mortality between D1 and D2 patients undergoing spleen resection only (Fig. 3a), or spleen and pancreas resection (Fig. 3b), no difference in mortality existed. When comparing hospital mortality within the D2 group only, all patients with their spleen/ pancreas preserved had significantly lower risk (RR 0.34, $p=0.02$ ) than their organ resection counterparts (Fig. 4c). This suggests that the higher overall hospital mortality reported in the D2 group of older trials was highly influenced by the spleen/pancreas resection.

Long-term outcomes

For the entire cohort of trials published, survival rates were similar irrespective of D1 or D2 surgical procedures (Fig. 6). These results correspond with those published in previous meta-analyses [19-21, 23, 24]. Although our subgroup analysis of patients with T3/T4 tumors did not find a significant survival benefit for D2 resections, a trend for improved survival does exist. The British trial performed by Edwards et al. [13] did conclude that there was a significant 5-year survival benefit for D2 resections in T3 patients (D2: $51 \%$ vs. D1: $17 \%, p=0.006$ ). This trial was not included in the present meta-analysis due to the confounding introduced by the randomization method to treatment arm by postal code. Additionally, our sub-analysis of spleen/pancreas-preserved patients revealed a trend whereby the 5-year survival was improved for D2 compared to D1 patients who did not have their spleen or pancreas removed ( $p=0.07$, Fig. 8).

The long-term benefit of the D2 procedure has gained merit with the publication of the 15-year follow up of the Dutch trial [8]. While overall 15-year survival was not significantly different between patients undergoing D1 $(21 \%)$ and D2 $(29 \%)$ resections $(p=0.34)$, the study confirmed the benefits of spleen/pancreas preservation [8]. Patients undergoing splenectomy and pancreatectomy had significantly lower overall survival in both D2 and D1 groups, whereas subgroup analysis of patients with spleen/ pancreas preservation showed a significantly higher overall 15-year survival for D2 patients (35\%) compared to D1 patients $(22 \%), p=0.006$ [8]. Thus, spleen/pancreas-preserving D2 resections appear to have the greatest benefit. The overall survival benefit was also apparent in stage II patients undergoing a D2 $(33 \%)$ versus D1 $(15 \%)$ resection $(p=0.03)$ [8]. Importantly, the gastric cancer-related death rate was significantly lower in the D2 group (37\%) 
compared to the D1 group ( $48 \% ; p=0.01)$, as was the local recurrence (D2: $12 \%$ vs. D1: $22 \%, p=0.015)$ rate [8]. Therefore, taking our meta-analysis together with the 15-year follow-up results of the Dutch trial, the long-term benefits of the D2 procedure cannot be ignored.

\section{Limitations and future directions}

While this is an updated meta-analysis comparing D1 and D2 resections, the 5-year survival results of the recent IGCSGR01 trial have yet to be published and thus its influence on the present results cannot be assessed. The major limitation of any meta-analysis of lymph node dissection trials is the change in protocol with respect to spleen and pancreas resection. This confounding variable may indeed be the reason behind a lack of significance in several outcomes. While we have conducted sub-analyses to highlight this protocol change, it can be argued that the three earlier trials and two recent trials are in fact not comparable, and that separate meta-analyses should be performed. With this in mind, and given that there are only 2 recent trials consisting of a very limited number of patients (D1: 243, D2: 245), reporting opposing results, we believe that it would be beneficial for one more, large, multi-center, D1 versus D2 RCT to be performed with the revised recommendations for spleen/pancreas preservation. An additional RCT may confirm the ability to perform D2 resections without significant postoperative increases in morbidity or mortality, as reported in the most recent trial. Furthermore, publication of longterm follow-up data from the Hong Kong and IGCSG-R01 trials is advocated to add to the robustness of the data concerning the survival benefits of D2 resections.

\section{Conclusion}

Surgery remains the most effective way to provide curative treatment for patients with gastric cancer. The debate between D1 and D2 resections has spanned from the 1980s to the present and has involved 5 RCTs (1642 patients: 845 D1, 797 D2). Improvements in surgical techniques and specifically organ preservation in the D2 procedure were employed in the most recent trial; however, these results cannot overpower those from the older, yet larger, trials that did not employ such techniques. When ignoring this confounding variable, overall hospital mortality and reoperation rates remained higher in D2 cases, despite the addition of the 2010 IGCSG-R01 trial results. However, when taking organ preservation into account, subgroup analysis revealed no significant difference in hospital mortality between D1 and D2 groups. Current available data demonstrated a lack of significance in overall 5-year survival rates with the D2 resection. However, a trend for improved survival was detected for T3/T4 patients, and for patients with spleen/pancreas preservation.

Acknowledgments This research is funded by the Canadian Cancer Society (grant \#019325). Dr. Coburn has received the Career Scientist Award through the Ontario Ministry of Health and Long-Term Care. Dr. Law is supported by the Hanna Family Chair in Surgical Oncology.

\section{References}

1. Hartgrink HH, Jansen EP, van Grieken NC, van de Velde CJ. Gastric cancer. Lancet. 2009;374(9688):477-90.

2. Gunderson LL, Sosin H. Adenocarcinoma of the stomach: areas of failure in a re-operation series (second or symptomatic look) clinicopathologic correlation and implications for adjuvant therapy. Int J Radiat Oncol Biol Phys. 1982;8(1):1-11.

3. Dent DM, Madden MV, Price SK. Randomized comparison of R1 and R2 gastrectomy for gastric carcinoma. Br J Surg. 1988; 75(2):110-2.

4. Cuschieri A, Fayers P, Fielding J, Craven J, Bancewicz J, Joypaul $\mathrm{V}$, et al. Postoperative morbidity and mortality after D1 and D2 resections for gastric cancer: preliminary results of the MRC randomised controlled surgical trial. The Surgical Cooperative Group. Lancet. 1996;347(9007):995-9.

5. Bonenkamp JJ, Songun I, Hermans J, Sasako M, Welvaart K, Plukker JT, et al. Randomised comparison of morbidity after D1 and D2 dissection for gastric cancer in 996 Dutch patients. Lancet. 1995;345(8952):745-8.

6. Cuschieri A, Weeden S, Fielding J, Bancewicz J, Craven J, Joypaul V, et al. Patient survival after D1 and D2 resections for gastric cancer: long-term results of the MRC randomized surgical trial. Surgical Co-operative Group. Br J Cancer. 1999;79(9-10): 1522-30.

7. Bonenkamp JJ, Hermans J, Sasako M, van de Velde CJ, Welvaart $\mathrm{K}$, Songun I, et al. Extended lymph-node dissection for gastric cancer. N Engl J Med. 1999;340(12):908-14.

8. Songun I, Putter H, Kranenbarg EM, Sasako M, van de Velde CJ. Surgical treatment of gastric cancer: 15-year follow-up results of the randomised nationwide Dutch D1D2 trial. Lancet Oncol. 2010;11(5):439-49.

9. Wu CW, Hsiung CA, Lo SS, Hsieh MC, Shia LT, Whang-Peng J. Randomized clinical trial of morbidity after D1 and D3 surgery for gastric cancer. Br J Surg. 2004;91(3):283-7.

10. Wu CW, Hsiung CA, Lo SS, Hsieh MC, Chen JH, Li AF, et al. Nodal dissection for patients with gastric cancer: a randomised controlled trial. Lancet Oncol. 2006;7(4):309-15.

11. Japanese Research Society for Gastric Cancer (JRSGC). Japanese classification of gastric cancer. 1st ed. Toyko: Kanehara; 1995.

12. Japanese Gastric Cancer Association (JGCA). Japanese classification of gastric carcinoma. 2nd ed. Gastric Cancer. 1998;1:10-24.

13. Edwards P, Blackshaw GR, Lewis WG, Barry JD, Allison MC, Jones DR. Prospective comparison of D1 vs modified D2 gastrectomy for carcinoma. Br J Cancer. 2004;90(10):1888-92.

14. Degiuli M, Sasako M, Ponti A. Morbidity and mortality in the Italian Gastric Cancer Study Group randomized clinical trial of D1 versus D2 resection for gastric cancer. $\mathrm{Br} \mathrm{J}$ Surg. 2010;97(5):643-9.

15. Schulz KF, Altman DG, Moher D. CONSORT 2010 statement: updated guidelines for reporting parallel group randomised trials. BMC Med. 2010;8:18. 
16. Hyde CJ, Stanworth SJ, Murphy MF. Can you see the wood for the trees? Making sense of forest plots in systematic reviews 2 . Analysis of the combined results from the included studies. Transfusion. 2008;48(4):580-3.

17. Hyde CJ, Stanworth SJ, Murphy MF. Can you see the wood for the trees? Making sense of forest plots in systematic reviews. Transfusion. 2008;48(2):218-20.

18. Hartgrink HH, van de Velde CJ, Putter H, Bonenkamp JJ, Klein Kranenbarg E, Songun I, et al. Extended lymph node dissection for gastric cancer: who may benefit? Final results of the randomized Dutch gastric cancer group trial. J Clin Oncol. 2004;22(11):2069-77.

19. McCulloch P, Nita ME, Kazi H, Gama-Rodrigues J. Extended versus limited lymph nodes dissection technique for adenocarcinoma of the stomach. Cochrane Database Syst Rev. 2003(4): CD001964.

20. Lustosa SA, Saconato H, Atallah AN, Lopes Filho Gde J, Matos D. Impact of extended lymphadenectomy on morbidity, mortality, recurrence and 5-year survival after gastrectomy for cancer. Meta-analysis of randomized clinical trials. Acta Cir Bras. 2008;23(6):520-30.

21. Yang SH, Zhang YC, Yang KH, Li YP, He XD, Tian JH, et al. An evidence-based medicine review of lymphadenectomy extent for gastric cancer. Am J Surg. 2009;197(2):246-51.
22. Tanizawa $\mathrm{Y}$, Terashima M. Lymph node dissection in the resection of gastric cancer: review of existing evidence. Gastric Cancer. 2010;13(3):137-48.

23. Memon MA, Subramanya MS, Khan S, Hossain MB, Osland E, Memon B. Meta-analysis of D1 versus D2 gastrectomy for gastric adenocarcinoma. Ann Surg. 2011;253:900-11.

24. McCulloch P, Niita ME, Kazi H, Gama-Rodrigues JJ. Gastrectomy with extended lymphadenectomy for primary treatment of gastric cancer. Br J Surg. 2005;92(1):5-13.

25. Degiuli M, Sasako M, Calgaro M, Garino M, Rebecchi F, Mineccia M, et al. Morbidity and mortality after D1 and D2 gastrectomy for cancer: interim analysis of the Italian Gastric Cancer Study Group (IGCSG) randomised surgical trial. Eur J Surg Oncol. 2004;30(3):303-8.

26. Robertson CS, Chung SC, Woods SD, Griffin SM, Raimes SA, Lau JT, et al. A prospective randomized trial comparing R1 subtotal gastrectomy with R3 total gastrectomy for antral cancer. Ann Surg. 1994;220(2):176-82.

27. Maruyama K, Sasako M, Kinoshita T, Sano T, Katai H, Okajima K. Pancreas-preserving total gastrectomy for proximal gastric cancer. World J Surg. 1995;19(4):532-6. 